\title{
A BIVARIATE OPTIMUM MODEL ON RECRUITMENT AND PROMOTION BASED ON EFFICIENCY AND SENIORITY
}

\author{
*M.Jeeva and **Rajalakhsmi Rajagopal
}

\begin{abstract}
In Manpower planning quantifative models have been used for predictingthe future structure and cost associated with it, given the current structure and policies on promotion, recruitment and wastage. In this paper a bivariate model has been discussed on fresh recruitments and promotions based on efficiency and seniority with the assumption on the maintainability of grade structure.

Key Words: Bivariate distribution, Promotion, Recruitment, Efficiency and Seniority
\end{abstract}

\section{Introduction}

Vacancies in any grade of a firm are filled either with promotions from next lower grade or by fresh recruitments. Promotion can be classified into two categories namely promotion based on efficiency and promotion based on seniority. Similarly recruitment can also be classified into the above two categories in the sense that seniority in recruitment means the equivalent work experience of a person in other firms and efficiency in recruitment is referred in the same sense as it is used in

* Lecturer, Department of Mathematics, Ethiraj College for Women, Chennai 600008 e-mail: mv_jeeva@yohoo.com.

* Head of the Department of Mathematics, Loyola College, Chennai - 600034 
promotion (such as possessing qualifications, training etc. to fulfil the needs of the management). Here we assume that promotion to a grade is allowed only from the

next lower grade. But persons from other lower grades with high efficiency in the same firm can compete as new recruits.

In this paper the above two aspects seniority and efficiency are considered in a bivariate framework both in promotion and in recruitment. First we apply the traditional Markov model to derive the estimates of the transition probabilities and policies towards promotions and recruitments under certain assumptions as in [2] with a difference of maintainability of grade structure over a finite period of time. Secondly we obtain the minimum levels of seniority and efficiency both in promotions and in recruitments and also obtain the corresponding cost associated with the above two operations.

\section{Notations}

$$
\begin{aligned}
& f=1,2, \ldots ., \text { T, T being the horizon usually ' } t \text { ' represents a year } \\
& i, i=1,2, \ldots \ldots, k \text { States of the system representing the various grades, total } \\
& N_{j}(t)=\text { Number of staff in grade } j \text { at the beginning of period } t \\
& p_{i j}(t)=\text { Probability that a member of staff in grade } i \text { at the beginning of period } \\
& t \text { is in grade } j \text { at the beginning of the next time period }(t+1) \\
& R_{i}(t)=\text { Number of new recruits to grade } j \text { during period } t: \\
& w_{i}(t)=\text { Wastage factor expressed as a proportion of members of staff of grade } i \\
& \mathrm{e}_{\mathrm{i}}=\text { Proportion of staff promoted from grade }(j-1) \text { to } j \text { : } \\
& 1-e_{i}=\text { Proportion of newly recruited staff to grade } i \text {. }
\end{aligned}
$$

\section{Assumptions}

1. No double promotion

2. No demotion

3. States of the system are mutually exclusive

4. $N(1)$, the vector of existing staff structure is known and $N(t)$, the vector of staff requirements for the future periods are assumed to be the same over a finite period of time $\mathrm{T}, t=1,2,3, \ldots, \mathrm{T}$ 
5. $w(t)$, the wastage vectors are known $\forall t=1,2, \ldots ., T$

4. Work Model

If $f=1$ represents the present period, then $N_{i}(1)$ for various values of $j$ represents the existing staff structure. Then under certain Markovian assumptions:we have [1]

$N_{j}(\mathrm{t}+1)=\sum_{i=1}^{k} \mathrm{p}_{\mathrm{ij}}(t) \mathrm{N}_{\mathrm{i}}(t)+\mathrm{R}_{\mathrm{j}}(t) \quad \forall \mathrm{j}=1,2 \ldots ., k$

Since at any point of time a member of the staff would either stay in the same grade, move to another grade or leave the system, we have

$\sum_{\mathrm{j}=1}^{\mathrm{k}} P_{\mathrm{i}}(t)+\omega_{\mathrm{i}}(t)=1 \quad \forall \mathrm{i}=1,2 \ldots, k$

As there are no double promotions and demotions along with the maintainability of grade structure over a period of time T, equations (1) and (2) takes the form

$N_{j}(t+1)=N_{j}(t)=P_{j j}(t) N_{j}(t)+p_{(j \cdot j] j}(t) N_{j, l}(t)+R_{j}(t)$

$P_{i j}(t)+p_{j(j+1)}(t)+\omega_{j}(t)=1 \quad \forall j=1,2 \ldots ., k$

With the above assumptions, the number of staff to be promoted and the number to be recruited for various grades can be estimated as follows.

For $t=1$ and $i=k$ (the highest grade) equations (3) and (4) become

$$
\begin{aligned}
& N_{k}(2)=N_{k}(1)=P_{k k}(1) N_{k}(1)+P_{(k-1) k}(1) N_{k \cdot 1}(1)+R_{k}(1) \\
& P_{k k}(1)=i-\omega_{k}(1) .
\end{aligned}
$$

(as there is no promotion from the highest grade, $p_{k(k+1)}(1)=0$ )

Therefore the total number of promotions and recruitments is given from equations (5) and (6) as

$$
P_{(k-l) k}(1) N_{k \cdot l}(I)+R_{k}(l)=N_{k}(l)-N_{k}(I)\left[I-\omega_{k}(1)\right]=N_{k}(1) \omega_{k}(1)=N_{k}(2) \text { (say) }
$$

since number of promotions and recruitments follow the ratio $e_{k}:\left(1-e_{k}\right)$ we have

$$
\begin{aligned}
& p_{(k-1) k}(1) N_{k-1}(1)=e_{k} N_{k}^{\prime}(2) \\
& R k(1)=\left(1-e_{k}\right) N_{k}^{\prime}(2)
\end{aligned}
$$

Equations (8) and (9) give the number of promotions from $(k-1)$ to $k$ and the number of new recruits to grade $k$ respectively. From equation (8) we have 
$P_{(K-1) k}(1)=\frac{e_{k} N_{k}^{\prime}(2)}{N_{k \cdot j}(1)}$

For $f=1$ and $j=(k-1)$ in $(4)$ we have

$p_{\{k-1)(k-1)\}}(1)=1-{ }_{w k-1}(1)-p_{(k-1) k}(1)$

By proceeding thus, the numbers of promotions and recruitments and the transitional probabilities can be estimated for all other states of the system at various time points.

\section{Bivariate framework}

Let $X$ and $Y$ be discrete random variables representing seniority and efficiency respectively.

Let $p_{1}(x, y)$ be the joint probability distribution of these two variables for all members of staff in grade $j$ in the organization and $F_{1}(x ; y)$ be the cumulative joint probability that $X \leq x$ and $Y \leq y$ :

Let $g_{I}(x)=\Sigma_{y} p_{j}(x, y)$ and $h_{j}(y)=\sum_{x} P_{j}(x, y)$ be the respective marginal distributions. Let the corresponding cumulative distribution functions be

$G_{i}(x)=P[X<x]$ and $H_{j}(y)=P\left[Y_{\leq} y\right]$

Suppose organization's policy exists for the proportion of promotions and recruitments based on seniority and on efficiency, then the respective minimum levels of these factors required for promotion and recruitment can be estimated.

During the first year $s_{i} N_{j} N_{i}(2)$ and $\left(1-s_{j}\right) e_{i} N_{j}^{\prime}(2)$ would be the number of promotes from grade $(j-1)$ to $i$ based on seniority and efficiency respectively, where $s_{i}$ and $(1-s$ ) are the proportions of promotions from grade $(j-1)$ to grade $i$ based on seniority and efficiency respectively. Similarly if $r_{i}$ and $(1-r)$ are the proportions of recruits to grade $j$ based on seniority and efficiency respectively, then $r_{i}\left(1-e_{j}\right) N^{\prime}(2)$ and $\left(1-r_{j}\right)\left(1-e_{j}\right) N_{i}(2)$ would be the number of recruitments in the grade $j$ based on seniority and efficiency respectively. If $x_{1}, x_{2}$ are the minimum levels of seniority required for promotion from $(j-1)$ to $j$ and recruitment into grade $j$ respectively, then they can be obtained from the following equations

$$
\begin{aligned}
& s_{j} e_{j} N_{j}^{\prime}(t+1)=N_{j-l}(t)\left[1-G_{j-1}\left(x_{j}\right)\right] \\
& r_{j}\left(1-e_{j}\right) N_{j}^{\prime}(t+1)=M_{j}(t)\left[1-G_{j}\left(x_{2}\right)\right]
\end{aligned}
$$

where $M_{i}(t)$ is total number of candidates appeared the interview for recruitmentinto grade $j$ during the time $f$. 
Similarly if $y_{1}, y_{2}$ are the minimum levels of efficiency required for promotion from grade $(i-1)$ to $j$ and recruitment into grade $j$; then we have

$$
\begin{aligned}
& \left(1-s_{j}\right) e_{j} N_{j}^{\prime}(t+1)=N_{j-l}(t)\left[1-H_{j-l}\left(y_{j}\right)\right] \\
& \left(1-r_{j}\right)\left(1-e_{j}\right) N_{j}^{\prime}(t+1)=M_{j}(t)\left[1-H_{j}\left(y_{2}\right)\right]
\end{aligned}
$$

The Order in which the promotion and recruitments based on these two factorsare to be given, may also influence the chance of a specific member of staff getting promoted or recruited. It does not affect the person with high values $X$ and $Y$ it is likely to affect those around the cut-off values of $X$ and $Y$ [ see equations (12) to (15)] These cut-off values are influenced by the degree of correlation between $X$ and $Y$.

Having got the number of promotions and recruitment from various cells of bivariate table the optimal cost can be obtained provided we are given the corresponding associated costs.

\section{Example}

Assume that a firm has only 3 grades with the following data.

$N(1)=\left(\begin{array}{l}470 \\ 335 \\ 280\end{array}\right)=N(2), \quad \omega(1)=\left(\begin{array}{c}0.2 \\ 0.1 \\ 0.05\end{array}\right)$

where $N(1) ; N(2)$ are the staff structure in three grades at time $f=1$ and $t=2$ respectively and $\omega(1)$ is the wastage rate at time $f=1$ from $N(1)$ during the first time period. Promotions from grade 1 to 2 and 2 to 3 are alone considered. The joint distribution of seniority and efficiency of staff in grade 1 and 2 are given in tables I and II. Also given are the following, $e_{2}=0.6, e_{3}=0.65, s_{2}=0.65$, $s_{3}=0.7, r_{2}=0.6$.

Table-I Distribution of Seniority and Efficiency of staff in grade 1 at time $\mathrm{t}=1$

\begin{tabular}{|l|c|c|c|c|}
\hline $\mathrm{nX}$ & $\leq 2$ & $(2,5)$ & $\geq 5$ & Total \\
\hline 1 & 100 & 70 & 40 & 210 \\
\hline 2 & 80 & 50 & 25 & 155 \\
\hline 3 & 60 & 35 & 10 & 105 \\
\hline Total & 240 & 155 & 75 & 470 \\
\hline
\end{tabular}


Table-ll Distribution of Seniority and Efficiency of staff in grade 2 at time $+=1$

\begin{tabular}{|l|c|c|c|c|}
\hline$n X$ & $\leq 2$ & $(2,5)$ & $\geq 5$ & Total \\
\hline 1 & 80 & 55 & 25 & 160 \\
\hline 2 & 60 & 30 & 15 & 105 \\
\hline 3 & 40 & 20 & 10 & 70 \\
\hline Total & 180 & 105 & 50 & 335 \\
\hline
\end{tabular}

Using equations from (3) to (11) in work model for this example recruitment vector $R(1)$ and the transition matrix $P(1)$ are obtained as follows

$$
R(1)=\left(\begin{array}{c}
120 \\
17 \\
5
\end{array}\right) \quad P(1)=\left(\begin{array}{lll}
0.745 & 0.055 & 0 \\
0 & 0.873 & 0.027 \\
0 & 0 & 0.95
\end{array}\right)
$$

This implies that for maintaining the staff grade structure at $f=1,2,5.5 \%$ of the staff from grade 1 and $2.7 \%$ of the staff from grade 2 should be promoted to the respective higher grades.

\section{Estimation of cut-off levels for seniority and efficiency for promotion}

The minimum (cut-off) level for promotion based on seniority and efficiency from grade 1 to 2 can be respectively obtained as follows.

From equation (7), $\mathrm{N}_{2}{ }_{2}(2)=43$. Number of promotions and recruitments into to grade 2 are calculated respectively as 26 and 17 using equations (8) and (9). From equation (12), we have,

that is

$$
(0.65)(0.6)(43)=470\left[1-G_{1}\left(x_{1}\right)\right]
$$

$$
17=470 P\left[X \geq x_{1}\right]
$$

This implies that out of 26 employees promoted, 17 are by seniority and the remaining 9 are by efficiency. From table $I$, we see that, the cell $[X \geq 5, Y=3]=$ 
10 , the remaining 7 are selected from cell $(X \geq 5, Y=2)=25$ by using simple interpolation as $7 / 25=0.28$. Therefore the cut-off level for promotion under seniority is $(X \geq 5, Y \geq 2.72)$. The balance 9 should be promoted under efficiency. They are selected from the cell $(X=(2,5), Y=3)=35$. Again by interpolation $[(9 \times 3=35)=$ 0.77 , we have the cut-off level for promotion under efficiency as $(X \geq 4.23, Y=3)$.

In the same way the minimum (cut-off) level for promotion based on seniority and efficiency from grade 2 to 3 can be obtained respectively from table ll as follows.

We have $\mathrm{N}_{3}^{\prime}(2)=14$. Number of promotions from grade 2 to 3 and recruitments to grade 3 are respectively 9 and 5 . Out of 9 promoted from grade 2 to 3,6 members are promoted by Seniority and 3 are by efficiency. The cell $(X \geq 5, Y=3)$ has 10 employees, which is 1 greater than the required number 9 . So the minimum cut-off level in this case by both seniority and efficiency will be $(X \geq 5, Y=3)$ with one person left from the cell. That person will be one with the minimum seniority among the 10 present in the cell. If by chance, all the 10 present in the cell possess the same seniority, then a new test has to be applied to select 9 persons among the 10 .

If costs associated with seniority and efficiency for promotions from grade 1 into grade 2 are given as

Table- III The cost associated with Promotion from grade 1 to grade 2

\begin{tabular}{|l|c|c|c|c|}
\hline $\mathrm{nX}$ & $\leq 2$ & $(2,5)$ & $\geq 5$ & Total \\
\hline 1 & 300 & 400 & 800 & 1500 \\
\hline 2 & 450 & 600 & 950 & 2000 \\
\hline 3 & 500 & 850 & 1200 & 2550 \\
\hline Total & 1250 & 1850 & 2950 & 6050 \\
\hline
\end{tabular}

The distribution of promotion from grade 1 into grade 2 is as follows.

Table-IV Distribution table of Promotion from grade 1 to grade 2

\begin{tabular}{|l|c|c|c|c|}
\hline $\mathrm{YX}$ & $\leq 2$ & $(2,5)$ & $\geq 5$ & Total \\
\hline 1 & 0 & 0 & 0 & 0 \\
\hline 2 & 0 & 0 & 7 & 7 \\
\hline 3 & 0 & 9 & 10 & 19 \\
\hline Total & 0 & 9 & 17 & 26 \\
\hline
\end{tabular}

Hence the optimal cost will be $7 \times 950+10 \times 1200+9 \times 850=$ Rs. 26300 


\section{Estimation of cut-off levels for seniority and efficiency in Recruitment}

Suppose that we are given the following table

Table-V Distribution table of candidates appearing interview for Recruitment in grade 2

\begin{tabular}{|l|c|c|c|c|}
\hline$n X$ & $\leq 2$ & $(2,5)$ & $\geq 5$ & Total \\
\hline 1 & 40 & 20 & 7 & 67 \\
\hline 2 & 20 & 8 & 4 & 32 \\
\hline 3 & 15 & 12 & 2 & 29 \\
\hline Total & 75 & 40 & 13 & 128 \\
\hline
\end{tabular}

From $R(1)$ we see that 17 should be recruited in grade $2[10$ under seniority, 7 under efficiency, using equation (13) and (15) ] for which the minimum (cut-off) levels for seniority and efficiency are respectively obtained as $(X \geq 5, Y \geq 1.43)$ and $(X \geq 3.25, Y=3)$.

Therefore the distribution of recruitment is as follows.

Table-VI Distribution table of Recruitment in grade 2

\begin{tabular}{|l|c|c|c|c|}
\hline$Y X$ & $\leq 2$ & $(2,5)$ & $\geq 5$ & Total \\
\hline 1 & 0 & 0 & 4 & 4 \\
\hline 2 & 0 & 0 & 4 & 4 \\
\hline 3 & 0 & 7 & 2 & 9 \\
\hline Total & 0 & 7 & 10 & 17 \\
\hline
\end{tabular}

If costs associated with seniority and efficiency for recruitments into grade 2 are given as follows 
Table-VII The cost associated with Recruitment in grade 2

\begin{tabular}{|l|c|c|c|c|}
\hline$n X$ & $\leq 2$ & $(2,5)$ & $\geq 5$ & Total \\
\hline 1 & 500 & 700 & 1000 & 2200 \\
\hline 2 & 800 & 900 & 1200 & 2900 \\
\hline 3 & 900 & 1300 & 2000 & 4200 \\
\hline Total & 2200 & 2900 & 4200 & 9300 \\
\hline
\end{tabular}

Then the optimal cost will be $4 \times 1000+4 \times 1200+2 \times 2000+7 \times 1300=$ Rs. 21900

\section{Conclusion}

In this paper Markovian Model is embedded in a bivariate framework to generate promotion probabilities and recruitments. The bivariate aspect of seniority and effi ciency associated with promotion and recruitment is studied. Our approach is very much relevant to the present day situation as most of the organizations follow the dual criteria of seniority and efficiency in promotion and recruitment.

\section{References}

1. D.J.Bartholomew, (1978) Stochastic Models for Social Processes, Wiley, London

2. B.G Raghavendra,(1991) J.opl.Res Soc Vol 42, no-7 pp565-570.

3. S.C.Gupta V.K.Kapoor,Fundamentals of Mathematical Statistics, Sultan and Sons. 